\title{
Antimicrobial susceptibility of $H$. pylori isolated from suspected patient using stool sample at Malali Hospital and Maternity Clinic Kaduna, Kaduna State - Nigeria
}

\author{
Rabilu Sani * \\ Department of Community Health Science, Shehu Idris Collage of Health Sciences and Technology, Makarfi, Kaduna State, \\ Kaduna - Nigeria.
}

GSC Biological and Pharmaceutical Sciences, 2021, 14(03), 122-128

Publication history: Received on 15 January 2021; revised on 09 February 2021; accepted on 11 February 2021

Article DOI: https://doi.org/10.30574/gscbps.2021.14.3.0042

\begin{abstract}
The antimicrobial susceptibility of Helicobacter pylori isolated from suspected patient using stool sample at Beijing greatest college of health sciences and technology, Kaduna, demonstration clinic.

The analyses were carried out to further understand the intestinal environment condition of the sample sites. As it has been noted that: it is difficult of growing Helicobacter pylori in a broth, hence, no specific enrichment has been proposed.

Note: As everything present in the stomach would be found in the stools, hence there is no doubt that Helicobacter pylori can be eliminated via this route and successful culturing of Helicobacter pylori from the stool sample had been achieved. [4]. The stool was diluted to a $20 \% \mathrm{w} / \mathrm{v}$ solution of in phosphate buffered saline (PBS) and the suspension was sieved through a 250 um strainer before plating onto the selective media. The sample was passed through a series of dilution techniques and centrifugation before finally plating on to the deoxycholate citrate agar (selective) and incubates at $37^{\circ} \mathrm{C}$ under the microacrobic atmospheric oxygen concentration of $7-12 \%\left(\mathrm{O}_{2}\right)$ for four (4) days.
\end{abstract}

Across the cultured, the cultured plate there was marked abundant of bacterial cells with some colonies having the characteristics of Helicobacter pylori which later sub-cultured and isolated for antimicrobial test.

Keywords: Antimicrobial; susceptibility; Helicobacter pylori; Patient; Stool; Microaerobic; Dilution and centrifugation.

\section{Introduction}

Helicobacter pylori, the principal species of the genus Helicobacter is a common human pathogen, which is responsible for a variety of gastro-duodenal pathologies in both the developing and the developed countries in the world. It is the main cause of at least $90 \%$ duodenal ulcer and $70 \%$ of gastric ulcer [1].

The bacterium is a slow-growing gram negative, curved or S shape rod while viewed in vivo, it is measured $2.5-3.5 \mu \mathrm{m}$ long with a single tuft of multiple-polar flagella and characteristically terminal bulb [2].

The organisms does not form spores but under adverse condition undergoes transformation from spiral to coccoid morphology often accompanied by a loss of culturability on conventional media [3].

The organism is fastidious and cultivation in vitro that requires microaerophilic environment and complex media. The bacteriological culture of Helicobacter pylori is quite tedious, time consuming procedure [5].

\footnotetext{
${ }^{*}$ Corresponding author: Rabilu Sani

Department of Community Health Science, Shehu Idris College of Health Sciences and Technology, Makarfi, Kaduna State, Kaduna Nigeria.

Copyright $(2021$ Author(s) retain the copyright of this article. This article is published under the terms of the Creative Commons Attribution Liscense 4.0.
} 
Culture undoubtedly constitutes the most specific way to establish the diagnosis of $H$. pylori infection, though its sensitivity has been reported to vary greatly among laboratories. Even experienced laboratories recover the organism from only $50 \%$ to $70 \%$ of infected biopsies. However, studies have shown that detection by culture can be comparable with detection by PCR. Recovery from stool, saliva, and vomitus is even more difficult because of the heavy colonization by more organisms [2], [3].

Bacteriological culture is a tedious, time-consuming procedure, and unnecessary for the routine diagnosis of $H$. pylori infection because other noninvasive tests will detect evidence of the organism in most patients [1].

Notwithstanding, culture allows testing of the sensitivity of $H$. pylori to the agents used for its eradication, a factor important to the clinician for the effective management of gastrointestinal problems caused by the bacterium.

Culture has also made an important contribution to our understanding of disease pathogenesis by enabling the development of techniques for phenotyping and genotyping isolates of the bacterium and for studying its pathogenic mechanisms in vitro and in vivo [4].

\section{Material and methods}

\subsection{Sample Site}

Stool sample were collected directly from fresh stool of the patient suspected to have history of gastric and duodenal ulcer [3].

\subsection{Foecal Sample and Collection}

A stool sample were collected from fresh defecated stool using a metal rod and it was dropped into the sterilized and free from chemical stool container [3].

\subsection{Sources of growth media (deoxycholate citrate media) and growth factor (supplements = blood)}

The Deoxycholate citrate media and the growth supplements (i.e. selenite F and blood) were purchased from MarcoHospital, chemical and reagents suppliers limited, Kaduna, Nigeria. All other chemicals were of analytical reagents grade [1].

\subsection{Determination of stool Physico-Chemical Parametres}

Feces are composed of water, protein, undigested fats, polysaccharides, bacterial biomass, ash, and undigested food residues. The major elements in feces as a percentage of wet weight are oxygen $74 \%$, hydrogen $10 \%$, carbon $5 \%$, and nitrogen $0.7 \%$, including the hydrogen and oxygen present in the water fraction of the feces [2], [1].

Feces compose a median value of $75 \% \mathrm{H}_{2} \mathrm{O}(\mathrm{n}=47)$ with a range of $63-86 \%$ across mean values of studies, variation can be attributed to differences in fiber intake as non-degradable fiber absorbs more water in the colon; therefore, as shown in a study, those with vegetarian diets will have a higher moisture content of 78.9\%, whereas those who consume less fiber and more protein will have a lower moisture content of $72.6 \%(\mathrm{p}=.001)$. Fiber intake also affects transit time, which has been positively correlated $(r=0.4, p=.03)$ with \% dry matter, showing the shorter the intestinal transit time the higher the water content. Variation in moisture content has been shown to vary with age; elderly people were found to excrete the highest amount of water in excreta of all age groups. Further deviations from the median value can be caused by illness. The mean generation rate of fecal water $(n=47)$ is $0.1 \mathrm{~L} / \mathrm{cap} /$ day. Average $\mathrm{pH}$ values for fecal water have been recorded at $\mathrm{pH} 6.9$ with a range of $\mathrm{pH} 5.0-8.0$ [3].

\subsection{Microbial Culture}

Deoxycholate Citrate agar was used for my own analysis which is one of the recommended media for the isolation of $H$. pylori species from the clinical samples of stools. The Deoxycholate citrate agar constituted the following ingredients that make bacterial cells to grow simultaneously [2], [3].

\subsubsection{Ingredients of $D C A$}

Ingredients Gms / Litre

HI solids - 10.000

Proteose peptone - 10.000 
Lactose - 10.000

Sodium deoxycholate -5.000

Neutral red - 0.020

Sodium citrate - 20.000

Ferric ammonium citrate -2.000

Agar - 13.500

Final $\mathrm{pH}\left(\right.$ at $\left.25^{\circ} \mathrm{C}\right)-7.5 \pm 0.2$

**Formula adjusted, standardized to suit performance parameters [6].

\subsection{Media Preparation}

70.52 grams of DCA was suspended in $1000 \mathrm{ml}$ of purified / distilled water. It was heated to boiling to ensure that the medium was dissolved completely. The excessive heating was avoided as it is detrimental to the medium. The medium was cooled to $45-50^{\circ} \mathrm{C}$. However, the medium was mixed thoroughly and poured into a sterile petri plates [8], [9].

\subsubsection{Preparation of the culture media}

Successful culturing of $H$. pylori from stool has been conducted, but the method is not easily reproducible. $H$. pylori lack regulatory genes making its survival for long periods outside the gastric environment very poor. To successfully isolate the organism from stool samples, the stool has been diluted to a $20 \% \mathrm{w} / \mathrm{v}$ solution in phosphate-buffered saline (PBS) and the suspension sieved through a $250 \mu \mathrm{m}$ strainer before plating onto selective media. A slightly different procedure to recover $H$. pylori from feces has also been conducted. A fresh fecal sample was suspended in $0.1 \mathrm{~mol} / \mathrm{L}$ sodium phosphate buffer to a final fecal slurry concentration of $20 \% \mathrm{w} / \mathrm{v}$. The suspension was centrifuged at $15,000 \mathrm{~g}$ for 30 minutes and then it was re-suspended in the buffer and a second centrifugation was performed before plating onto the selective growth media [7].

\subsubsection{Inoculation}

The Deoxycholate citrate agar has been prepared (DCA) and it was poured into the sterile petri dish and the lid were replaced immediately and the agar plate was allowed to cool and set like gelatin at room temperature for certain period of time. However the inoculant has been inoculated in to the petri dish containing gelatin agar (DCA) and distributed evenly into the media [8].

\subsubsection{Incubation}

The plate was incubated under microaerobic conditions at $37^{\circ} \mathrm{C}$ for four (4) days at the oxygen concentration of $7-12 \%$ $\left(0_{2}\right)$ and this was achieved using atmospheric incubator and the colony formation was achieved sufficiently [9], [10].

\subsubsection{Identification of $H$. pylori}

The identification of cell morphology was done by Gram's stain reaction and positive biochemical reaction for catalase, Urease and Oxidase [6], [8].

\subsection{Characterization of $H$. pylori on DCA and biochemical reaction}

\subsubsection{Uresse}

The hydrolysis of urea was detected by streaking the culture on Christensen agar medium containing peptone beside urea and small amount of glucose, with phenol re as an indicator for 2 days [7].

\subsubsection{Catalase production}

The catalase test was performed directly on colonies growing on "Columbia agar" the hydrogen peroxide $\left(\mathrm{HO}_{2}\right)$ solution $(3 \%)$ was used to flood colonies. (+) [8], [10].

\subsubsection{Oxidase}

This was performed directly on the "colonies agar"; A 1\% aqueous tetra-methyl -P- Phenelyne diamine - dehydrochloride (TMPPD) impregnated paper on which a loop-full of young culture was streaked (+) [6], [10]. 


\subsubsection{Colony and cell mophology}

Experimentally, the overnight grown culture in DCA were spread on the petri dish containing the gelatin agar and incubated at $37^{\circ} \mathrm{C}$ for a good four (4) consecutive days, the colour of the cell colony was yellowish and however, the colour depend on the media. The morphology of the colonies was noted, the cell motility was as well noted and shape of the organisms was rod in shape and size of $0.5-1 \mathrm{~mm}$ of a single colony was observed under microscope [6].

\subsection{The antimicrobial susceptibility of $H$.pylori}

The phenotypic methods based on the disc diffusion was conducted for these research, in this research, the $H$. pylori were isolated and expressed an increasing resistance in respect to antimicrobial currently used in the therapy. The aim of this research is to evaluate the antimicrobial profile of $H$. pylori isolated from the stool sample.

The stool sample were taken from the suspected patient that has a positive test of occult analysis.

The antibacterial susceptibility was performed for metronidazole, levofloxacin, ciprofloxacin, tetracycline, amoxicillin and ampicillin respectively on modified agar susceptibility test [9].

\section{Results}

Table 1 The table showing the biochemical and morphological characteristics of H. Pylori [7], [10].

\begin{tabular}{|l|l|}
\hline Assay & Observation \\
\hline Colony shape & Round, small, translucent, $2-3 \mathrm{~mm}$ \\
\hline Cell shape & Spiral, helical or curved with blunt ends \\
\hline Cell motility & + \\
\hline Gram staining & - \\
\hline Urease & + \\
\hline Oxidase & + \\
\hline Catalase & + \\
\hline Nitrate reduction & - \\
\hline Glycine utilization & - \\
\hline Growth on Nutrient agar & - \\
\hline Columbia agar & + \\
\hline Brucell agar & + \\
\hline Blood agar & + \\
\hline Brain heart infusion agar & + \\
\hline Serum supplement agar & + \\
\hline Growth at & \\
$25^{\circ} \mathrm{C}$ & - \\
$30^{\circ} \mathrm{C}$ & - \\
$35^{\circ} \mathrm{C}$ & + \\
$40^{\circ} \mathrm{C}$ & + \\
$45^{\circ} \mathrm{C}$ & - \\
\hline Growth in NaCl (\%) & \\
0.5 & + \\
0.75 & + \\
1.0 & + \\
1.25 & + \\
1.5 & - \\
\hline
\end{tabular}




\begin{tabular}{|l|l|}
\hline Resistance/sensitivity $(50 \mu \mathrm{g} / \mathrm{ml})$ & \\
Clarithromycin & + \\
Metronidazole & + \\
Levofloxacin & - \\
Ciprofloxacin & - \\
Tetracycline & - \\
Amoxicillin & - \\
\hline
\end{tabular}

The antimicrobial susceptibility was successfully conducted. The percentages of the resistance were as follows:

Table 2 The table below shows the antimicrobial used and resistance percentage.

\begin{tabular}{|l|l|l|}
\hline Clarithromycin & - & $72.28 \%$ \\
\hline Metronidazole & - & $34.69 \%$ \\
\hline Levofloxacin & - & $42.85 \%$ \\
\hline Ciprofloxacin & - & $44.28 \%$ \\
\hline Tetracycline & - & $2.63 \%$ \\
\hline Amoxicillin & - & $1.02 \%$ \\
\hline Ampicillin & - & $0 \%$ \\
\hline
\end{tabular}

The table above shown that the research underlines the high rate of resistance to clarithromycin, metronidazole, and the quinolone which may reflects over used of them.

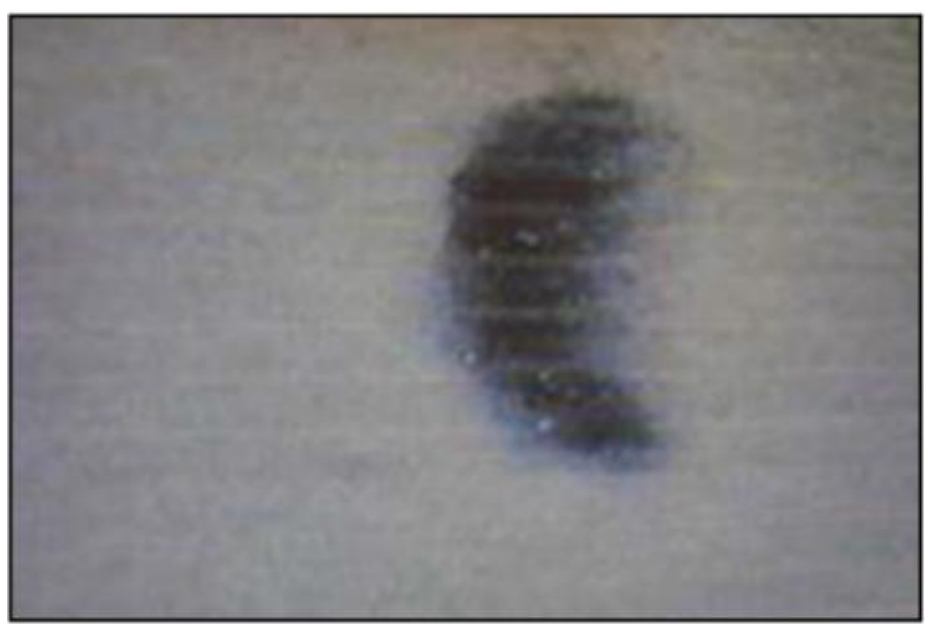

Figure 1 A diagram of $H$. pylori on a slide for gram staining. 


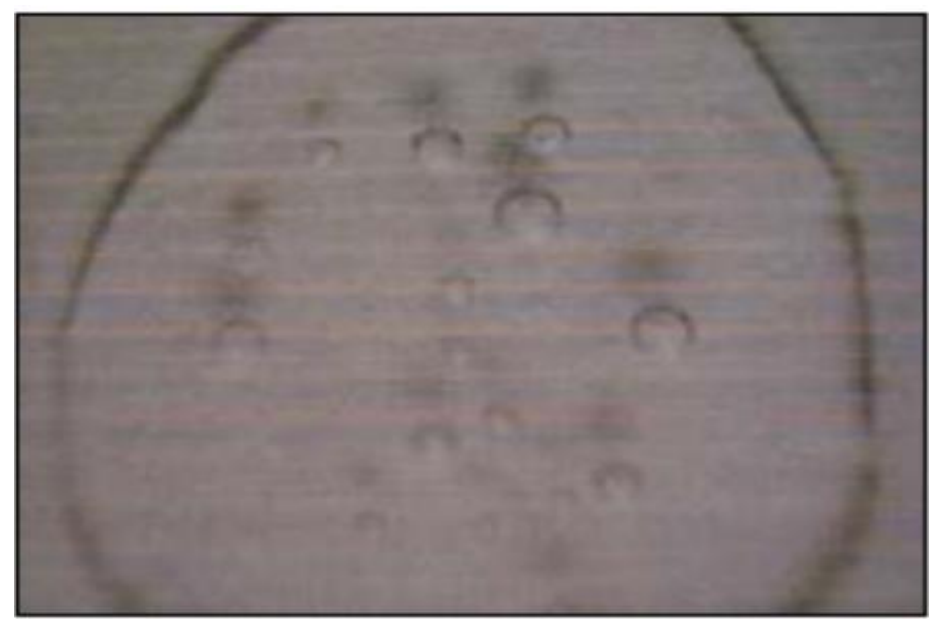

Figure 2 Detection of cell motility

\section{Discussion}

H. pylori infection is one of the most common infections worldwide.

However, it is not known how H. pylori are transmitted and wherein the natural environment the organism resides. It is likely that raw sheep's milk could be an intermediate transmission vehicle of $H$. pylori infection. It has been reported that $H$. pylori are almost always acquired in childhood. In other hand, reported that $H$. pylori were detected in cow's feces and soil and the bacteria could invade the teat channel of a cow when it was sprawled on the ground including cow's feces and soil. As reported that H. pylori gene is frequently detected in cow's milk samples, the samples might have been contaminated with the organism from contaminated soil. Furthermore, they have shown the possibility that $H$. pylori survive in raw milk. This study was investigated to isolate, identify, and found the total count of bacterial colony and occurrence in gastric patients [10].

The antibiotics resistance and sensitivities are an essential assay for the pathogen. In the present research, different kinds of antibiotics such as Tetracycline, Clarithromycin, Metronidazole, Levofloxacin, Ciprofloxacin, Amoxicillin and ampicillin were used. Then, the MIC was recorded. Among the tested antibiotics, the bacterium showed moderate sensitivity to above mentioned drugs [6], [8].

These resistant characters were also very much useful in selecting marker for classical genetic experiments.

In our present study, the isolated bacterium $H$. pylori showed only a moderate sensitivity against various tested antibiotic. Similarly, also found the resistance of $H$. pylori against clarithromycin. Hence, they successfully developed clarithromycin loaded mucoadhesive microspheres to completely eradicate the $H$. pylori in the stomach.

Gastric cancer is the second most common cause of cancer-related mortality worldwide. Detection of the disease usually occurs at an advanced stage and overall survival rates for gastric cancer are poor. The present model for gastric cancer progression clearly maintains Helicobacter infection as the primary inducer of gastric metaplastic and neoplastic disease. H. pylori are a ubiquitous organism, infecting more than half the world's population. It has been suggested that this infection directly contributes to the formation of gastric cancer in up to $80 \%$ of cases; however, gastric malignancy develops in only a subset $(<1 \%)$ of infected patients. Predisposition to Helicobacter associated gastric cancer is most likely multifactorial, including the interaction of bacterial, host and environmental components [8], [10], [6].

The development of intestinal-type gastric cancer is a multistep process. It involves temporal progression from chronic gastritis to gastric atrophy, intestinal metaplasia, dysplasia, and finally gastric cancer. While a number of factors contribute to this transition, it has become clear that H. pylori are the primary trigger for neoplastic progression. The association between $H$. pylori and gastric cancer has been known for over a decade. The early studies estimated the prevalence of $H$. pylori in patients with gastric cancer and recent work using a combination of two or three methods to diagnose $H$. pylori infection have shown a much stronger association [7], [9].

Childhood is the critical period for infection and transmission of disease. 
Most probably occurs from person to person. The iatrogenic route certainly exists but is considered relatively unimportant. Much debate surrounds the oral-oral and fecal-oral routes, which are probably more significant [6], [7].

\section{Conclusion}

At the end of this study, it has been observed that the human stomach and intestine were the only known reservour of the H. pylori. However, the possibility that there are other reservoures cannot be excluded, as the conditions required for growth were met in the gastro intestinal tract of all worm-blooded animals.

The result from various studies have shown that the Helicobacter species, but other Helicobacter species have been isolated from other animals, this suggested that there were presence of a host specific binding sites, although the techniques require for the isolation of Helicobacter species might differ between various hosts [8], [10].

\section{Compliance with ethical standards}

\section{Acknowledgments}

I wish to thank the laboratory staffs of the Malali Hospital and Maternity Clinic of this research.

\section{Disclosure of conflict of interest}

Virtually, there's no what so ever any conflict of interest, I am here giving the outfit full right to publication of the work.

\section{References}

[1] Argyros FC, Ghosh M, Huang L, et al. Evaluation of a PCR primer based on the isocitrate dehydrogenase gene for the detection of Helicobacter pylori infeces. J Clin Microbiol. 2000; 38: 3755-58.

[2] Mapstone NP. PCR identification of Helicobacter pylori in faeces from gastritis patients. Lancet. $1993 ; 341: 447$.

[3] Gramley WA, Asghar A, Frierson HF JP, et al. Detection of Helicobacter pylori DNA in faecal samples from infected individuals. J Clin Microbiol. 1999; 37: 2236-40.

[4] BSAC Working Party. Recommendations for susceptibility tests on fastidious organisms and those requiring special handling. J Antimicrob Chemother. 2001; 48 (suppl S1): 77-80.

[5] Megraud F, Hazell S, Glupczynski Y. Antibiotic susceptibility and resistance. In: Mobley HLT, Mendz GL, Hazell SL eds. Helicobacter pylori: physiology and genetics. Washington DC: ASM Press. 2001: 511-30.

[6] Isenberg RD. Clinical Microbiology Procedures Handbook 2' Edition.

[7] Jorgensen JH, Pfaller MA, Catroll KC, Funke G, Landrv ML, Richter SS, Wamock DW. Manual of Clinical Microbiology. 2015.

[8] Salfinger Y, Tortorello ML. Compendium of Methods for the Microbiological Examination of Foods. 5th Ed. American Public Health Association. Washington. D.C. 2015.

[9] Rupprecht HJ, Blankenberg S, Bickel C, et al. Impact of viral and bacterial infectious burden on long-term prognosis in patients with coronary artery disease. Circulation. 2001;104:25-31.

[10] Pietroiusti A, Diomedi M, Silvestrini M, et al. Cytotoxin-associated gene-A-positive Helicobacter pylori strains are associated with atherosclerotic stroke. Circulation. 2002;106:580-4. 